\title{
Modificaciones en los últimos escenarios sexuales, en los chicos
}

\author{
Modifications of the last sexual stages, of the boys \\ Antonio López-Castedo, María Isabel Nóvoa, Encarnación Sueiro \\ Fac. CC. de la Educación. (U.Vigo-Campus de Ourense)
}

\begin{abstract}
Resumen
Analizamos los cambios ocurridos en las últimas conductas coitales, en los hombres, durante un intervalo de 10 año, mediante un estudio longitudinal, diacrónico o transversal y de tendencia con una muestra de 2245 universitarios (curso 97-98) y 856 (07-08), que realizaban primero y último en la Universidad de Vigo. Se les aplicó una encuesta estructurada, anónima y voluntaria en la que se recogía la edad y variables sexuales, referidas a las últimas conductas coitales. Los datos se analizan mediante el SPSS 21.0. La edad media de las hombres universitarios, del curso 97-98, es de 20.85 años y la del curso 07-08, de 21.42 años. Las diferencias son significativa $(\mathrm{p}<.05)$ en cuanto a quienes iniciaron los coitos y edad, tiempo transcurrido desde el último coito, tipo de pareja sexual, satisfacción, por amor y deseo, el lugar donde aconteció, frecuencia coital, consumo etílico, uso de anticoncepción y elección de la misma.

Palabras clave: cambios, conductas coitales, universitarios
\end{abstract}

\begin{abstract}
In the last decades, sexuality is one of the facets that raises more interest among evolutionary researchers. In this research the change occurred in the noncoital behavior that are analyzed in males, for a period of 10 years. It took a very lengthy study, diachronic o transversal, with a trend over a sample of 2245 young university students (course 97 98) and 856 (course 07-08), in they were in their first and last year of their university course, in the University of Vigo. It was applied a structured survey, anonymous and voluntary, in which was the ages and variables were gathered, contemplating their noncoital behavior. The average age of university male, course 97-98, was of the ages $20-85$ years old and the course $07-08$, was of the ages 21-42 years old. The data was anaylzed using the statistical program SPSS 21.0.
\end{abstract}

Keywords: changes, coital, universitary males.

\section{Método}

Estudio longitudinal, diacrónico o transversal y de tendencia, desarrollado sobre una muestra de 2245 jóvenes universitarios que realizaban el primer y último curso, en el Campus de Vigo, de Ourense y de Pontevedra, de la Universidad Sur de Galicia, en el curso 1997-1998 y por 856 del curso 2007-2008., a quien se le aplicó una encuesta estructurada, anónima y voluntaria en la que se recogía la edad y variables referidas al último coito: su inicio y edad de comienzo, sexo de la última pareja, tipo de pareja, sentimientos experimentados, tiempo de conocimiento previo, razones que la motivaron, lugar en el que aconteció, frecuencia y número de parejas durante el último año, existencia de orgasmo, consumo etílico, uso de protección, tipo, existencia de problemas con su uso y quien la propone.

Los datos se analizan mediante el SPSS 21.0. para hallar la Media y Desviación Típica (S), para la descripción de las variables cuantitativas, y la frecuencia y porcentaje, para la descripción de las variables cualitativas. Para hallar las diferencias significativas, empleamos la prueba t y la Chi cuadrado.

\section{Resultados}

La edad media de los hombres universitarios del curso 97-98 es de 20.85 años (D.T.: 2.54) y la de los del curso 07-08, es de 21.42 años (D.T.: 2.91).

El 50.6\%, de los hombres, del curso 97-98, habían iniciado sus coitos, a una edad media de 18.00 años, lo mismo que el 78.3\%, de los del curso 07-08, a una edad media de 16.97 años. Encontramos diferencias significativas en cuanto al porcentaje de comienzo $\left(\chi^{2}=\right.$ $193.26 ; \mathrm{p}=0.000)$ y a la edad de comienzo $(\mathrm{t}=18.616$; $\mathrm{p}=0.000)$.

Los resultados que siguen hacen referencia sólo a quienes iniciaron los coitos.

El 37.0\% de quienes estudiaron en el curso 97-98 y el $45.4 \%$ de quienes lo hicieron en el 07-08 tuvieron su último coito hacía menos de 1 semana; un $20.9 \%$ de los primeros y un $22.2 \%$ de los segundos hacía más de 1 semana y menos de 1 mes; un $18.5 \%$ y un $13.9 \%$, respectivamente, entre 2 y 5 meses; mientras que los restantes $(22.4 \%$ y $14.8 \%$, respectivamente) hacia más de 6 meses. Hay diferencias significativas entre ambas muestras $\left(\chi^{2}=32.10 ; \mathrm{p}=0.000\right)$.

El $87.6 \%$ de los del curso $97-98$, y el $89.7 \%$ de los del 07-08, mantuvieron su último coito con una persona de diferente sexo; mientras que el $8.8 \%$ de los primeros y el $7.2 \%$ de los segundos, fue con una persona del mismo sexo, sin diferencias significativas $\left(\chi^{2}=2.27 ; p=0.320\right)$ entre ambos grupos .

El último coito fue con su pareja, para el $63.4 \%$ de los primeros y el $53.9 \%$ de los segundos; mientras que para un $26.9 \%$ de los primeros y un $29.9 \%$ de los segundos, con una amiga, y para un $8.0 \%$ y un $14.7 \%$, respectivamente, con una prostituta, esposa u otras. Hay diferencias significativas entre ambos grupos $\left(\chi^{2}=\right.$ $37.67 ; \mathrm{p}=0.000)$. 
El 78.6\% de los varones del curso 97-98, y el $87.5 \%$ del curso $07-08$, se sintieron satisfechos en la última relación coital; mientras un $17.7 \%$ de los primeros y un $10.2 \%$ de la segunda, se sintieron indiferentes, insatisfechos, mal, preocupados u otra sensación. Existen diferencias significativas $\left(\chi^{2}=24.73 ; p=0.000\right)$ entre ambos grupos.

Hacia más de 1 año que conocían a la persona con quien tuvieron su último coito el $57.4 \%$ de los primeros y el $51.6 \%$ de los segundos; un $22.8 \%$ y un $26.1 \%$, respectivamente, hacia más de 1 mes, pero menos de 1 año; y un $17.9 \%$ y $19.8 \%$, respectivamente, menos de 1 mes, no encontrándose diferencias significativas entre ambos grupos $\left(\chi^{2}=9.10 ; \mathrm{p}=0.058\right)$.

Un $75.4 \%$ del curso $97-98$, y un $69.4 \%$ del curso $07-$ 08 , tuvieron el último coito porque sentían deseo; un $41.5 \%$ y un $35,7 \%$, respectivamente, porque estaban enamorados; mientras que los demás motivos estudiados (sentía curiosidad, temor a perder a mi pareja, presionado por mi mi pareja, por mi grupo de amistades y deseaba tener un hijo) fueron muy poco elegidas por los varones de ambas muestras. Las diferencias son significativas entre los hombres de ambos grupos en: estar enamorado $\left(\chi^{2}=5.02 ; p=0.023\right)$, sentir deseo $\left(\chi^{2}\right.$ $=6.75 ; \mathrm{p}=0.009)$ y desear tener un hijo-a $\left(\chi^{2}=4.75 ; \mathrm{p}\right.$ $=0.029) ;$ sin embargo, no lo son en: tenía curiosidad $\left(\chi^{2}\right.$ $=0.872 ; \mathrm{p}=0.350)$, temor a perder a mi pareja $\left(\chi^{2}=\right.$ $3.43 ; \mathrm{p}=0.064)$, presionada por mi pareja $\left(\chi^{2}=0.61 ; \mathrm{p}\right.$ $=0.433)$ y presionada-o por mi grupo de amistades $\left(\chi^{2}=\right.$ $0.08 ; \mathrm{p}=0.776)$.

El $43.0 \%$ y el $13.8 \%$ de los primeros y el $49.9 \%$ y el $17.5 \%$ de los segundos, realizaron su último coito en casa propia y prestada, respectivamente; un $20.5 \%$ y un $14.0 \%$, respectivamente, en el coche; mientras que, los otros lugares elegidos (hotel $/ \mathrm{motel} /$ pensión, espacios públicos u otros), daban un $20.7 \%$ para la primera muestra y un $16.6 \%$ para la segunda. Hallamos diferencias significativas $\left(\chi^{2}=25.21 ; \mathrm{p}=0.000\right)$ entre ambos grupos.

El $35.8 \%$ de los varones del curso $97-98$ y el $27.9 \%$ del curso 07-08 mantuvieron una frecuencia coital de menos de $1 \mathrm{vez} / \mathrm{mes}$; el $29.6 \%$ de la primera muestra y el $40.5 \%$ de la segunda, más de 1 vez/semana. Se encuentran porcentajes similares en ambos grupos para el intervalo de 1 a 3 veces al mes (en torno al 28.0\%). Hay diferencias significativas entre ambas muestras $\left(\chi^{2}\right.$ $=32.16 ; \mathrm{p}=0.000)$.

El $64.3 \%$ de quienes cursaron en $97-98$ y el $62.5 \%$ de quienes lo hicieron en 07-08 tuvieron una única pareja coital; el $12.3 \%$ de los primeros y el $14.3 \%$ de los segundos, tuvieron dos y el $16.4 \%$ y el $15.5 \%$, respectivamente, tres o más parejas, no siendo la diferencia no es significativa entre ambos grupos $\left(\chi^{2}=\right.$ 2.97; $\mathrm{p}=0.562$ ).

El $86.4 \%$ del curso $97-98$, y el $84.2 \%$ del curso $07-08$, sintieron orgasmo en el último coito; por el contrario, el $9.0 \%$ de los primeros y el $10.1 \%$ de los segundos, no, no encontrando diferencias significativas entre ambos $\left(\chi^{2}=\right.$ $0.80 ; \mathrm{p}=0.370)$.

El $69.1 \%$ de los chicos del curso $97-98$, y el $64.9 \%$ del curso $07-08$, no consumieron alcohol en el momento del último coito; mientras que, un $27.8 \%$ de los primeros y un $32.4 \%$ de los segundos, sí, existiendo diferencias significativas entre ambos grupos $\left(\chi^{2}=4.06\right.$; $\mathrm{p}=0.044)$.

El $84.9 \%$ del curso $97-98$ y el $86.9 \%$ del curso $07-08$, usaron algún método de protección en su último coito; mientras que un $11.3 \%$ de los primeros y un $8.1 \%$ de los segundos, no, hallando diferencias significativas $\left(\chi^{2}=\right.$ $4.45 ; \mathrm{p}=0.035)$ entre ambos. El $81.7 \%$ de los varones del curso $97-98$ y el $79.4 \%$ del curso $07-08$ usaron el preservativo; un $11.3 \%$ de los primeros y un $15.3 \%$ de los segundos, los hormonales, y un $2.4 \%$ y un $1.7 \%$, respectivamente, el coito interrumpido. Los restantes métodos anticonceptivos (natural, espermicida, esponja, diafragma y D.I.U.) apenas fueron empleados por los varones de ambos grupos, no encontrando diferencias significativas $\left(\chi^{2}=9.97 ; \mathrm{p}=0.190\right)$.

E1 $94.1 \%$ de los chicos del curso $97-98$ y el $93.1 \%$ del curso 07-08 no tuvieron problemas con el último método anticonceptivo empleado; sin embargo, un 5.3\% de los primeros y un $4.5 \%$ de los segundos, sí, no encontrándose diferencias significativas entre los varones de ambos cursos $\left(\chi^{2}=0.41 ; \mathrm{p}=0.520\right)$.

Finalmente, el $68.8 \%$ del curso $97-98$, y el $70.3 \%$ del curso 07-08, manifestaron que la propuesta del último método de protección fue propia; sin embargo, para un $17.1 \%$ de los primeros y un $21.6 \%$ de los segundos, fue de su pareja; y un $11.7 \%$ y un $5.3 \%$, respectivamente, lo propusieron ambos. La diferencias son significativas $\left(\chi^{2}\right.$ $=70.35 ; \mathrm{p}=0.000)$ entre ambos grupos de chicos.

\section{Discusión}

Siendo el 50.6\% (97-98) y el 78.3\% (07-08) los chicos que iniciaron sus coitos a las edades de 18 y casi 17 años, respectivamente, encontramos que el 37.0\%, de los hombres del curso 1997-98, y el 55.4\%, del curso 2007-08, afirmaron que su último coito se produjo hacía menos de 1 semana, y un $57.9 \%$, de los primeros, y un $77.6 \%$, de los segundos, hacia menos de 1 mes, poniéndose de manifiesto cambios significativos después de 10 años. De esta manera, aunque los estudios relacionados con esta variable no son totalmente comparables ya que se fijan en otro tipo de intervalos, en la investigación de Ochaita y Espinosa (2003), sobre jóvenes urbanos, afirman que un $20.4 \%$ de los chicos no han tenido coitos en este último mes, el $20.3 \%$ entre 1 y 4 veces; un $12.1 \%$ entre 5 a 8 veces, el $15.2 \%$ de 9 a 16 veces, un $4.8 \%$ más de 16 veces, y el $27.2 \%$ no contestaron a la pregunta, porcentajes inferiores a la muestra de varones del curso 2007-08 sumando los jóvenes que tuvieron coito en el último mes $(52.4 \%)$. 
La 2a Encuesta Schering de Sexualidad y Anticoncepción en la Juventud Española (2005) constata que el $81 \%$ han mantenido relaciones coitales en los últimos 12 meses y el número de relaciones sexuales al mes en el último año es de 8.4 , porcentajes inferiores a los obtenidos con los del curso 2007-08. A su vez, Failde et al. (2008), aclaran que un $66.4 \%$ de los chicos han tenido relaciones sexuales en los últimos 6 meses.

Atendiendo al sexo de la persona con que tuvo el último coito, se observa que el $87.6 \%$, de los varones del curso 1997-98, y el 89.7\%, del curso 2007-08, manifiestan que su último coito lo practicaron con una persona de diferente sexo, y el $8.8 \%$, de la primera muestra, y el $7.2 \%$, de la segunda, con parejas sexuales del mismo sexo. Aunque la mayoría de las investigaciones se centran en la orientación sexual de la persona y no tanto con quien tuvieron la última relación coital, Ochaita y Espinosa (2003), mencionan que el $3.5 \%$ de los hombres jóvenes urbanos mantienen coitos con personas del mismo sexo y un $0.6 \%$ indistintamente, con un porcentaje de varones que no contestan $(34.5 \%)$, poniéndose de manifiesto resultados inferiores a los encontrados en los varones del curso 2007-08, siendo debido a que en nuestra investigación sólo se tiene encuesta el último coito.

Analizando el estudio del IJE-2008, se advierte que la tasa global de homosexualidad y de bisexualidad reconocida entre los y las jóvenes en España se sitúa en el $4.0 \%$. Si se compara los distintos IJEs desde el año 2000, las tasas de homosexualidad se van incrementando, pasando de un $1.6 \%$, para los varones en el año 2000 , a el $3.5 \%$, para los varones en el año 2008, situándose las máximas en el año 2002, con un $5.3 \%$ (Comas, 2003), reafirmando los datos de la muestra de varones del curso 07-08, y reflejando no tanto un crecimiento de la homosexualidad, sino una disminución del grado de ocultamiento de su condición sexual por parte de los jóvenes (Comas, 2008).

Si nos centramos en la relación existente con la última pareja sexual, en ambas muestras, se expresa que fue con la novio-a, aunque los porcentajes decrecen significativamente del curso 1997-98 (63.4\%) al 200708 (53.9\%), consecuentemente, se incrementó de manera significativa, el porcentaje de personas que lo realizaron con una amistad o con otras personas.

A su vez, Lameiras et al. (2007-08), afirman que, los chicos sexualmente activos, el $41.7 \%$ mantienen relaciones sexuales con una pareja estable, y un $12.5 \%$ con diferentes personas..

Respecto a la variable de cómo se sintieron en la última relación coital, se pone de manifiesto que un $78.6 \%$, de los hombres del curso 1997-98, se sintieron satisfechos-as, incrementándose este porcentaje significativamente a un $87.5 \%$, para los hombres del curso 2007-08, encontrando cambios significativos después de 10 años de evolución.
Sin embargo, Rodríguez y Traverso (2007), manifiestan que la valoración del sentimiento en las relaciones coitales en el momento de cumplimentar la encuesta, es del $82.6 \%$ satisfactorio o muy satisfactorio, en un $8.2 \%$ insatisfactorias o muy insatisfactorias, y en el $9.2 \%$ de los hombres la relación no dejó especial huella (ni satisfactoria ni insatisfactoria), resultados que concuerdan totalmente con los encontrados en los varones del curso 2007-08.

En relación al tiempo que hacía que conocía a la persona con quién aconteció esa última relación sexual coital, para la muestra del curso 1997-98, el porcentaje más alto $(57.4 \%)$, se encuentra en el intervalo de 1 año o más tiempo, que desciende al $51.6 \%$, para los del curso 2007-08, sin encontrar cambios significativos después de 10 años.

En cuanto a las razones por las que realizaron ese último coito, en ambas muestras, se pone de manifiesto que es en el deseo donde se adhiere el mayor porcentaje de sujetos $(75.4 \%$, para la primera, y $69.4 \%$ para la segunda), seguida del amor (41.5\% y $35.7 \%$, respectivamente), siendo en ambos casos el descenso significativo entre ambas muestras.

Por lo que se refiere al lugar donde ocurrió la última relación coital, se observa que fue la casa propia o prestada (del $56.8 \%$, de la primera muestra, se pasa al $67.4 \%$, de la segunda), seguido del coche $(20.5 \%$ y $14.0 \%$, respectivamente), y el espacio público con el $7.7 \%$, para el curso $1997-98$ y el $7.5 \%$, para el $2007-08$, encontrándose cambios significativos a lo largo de los diez años.

$\mathrm{Si}$ analizamos la variable sobre la frecuencia con que aconteció el coito durante el último año, se observa que los mayores porcentajes, en ambos cursos, se encuentran en el intervalo de menos de $1 \mathrm{vez}$ al mes (el $35.8 \%$, del alumnado del curso $1997-98$, y $27.9 \%$, del curso 2007-08), seguido del intervalo de 1 a 3 veces al mes, poniéndose de manifiesto diferencias significativas con el paso del tiempo. Así, la $1^{\mathrm{a}}$ encuesta de Salud y Sexualidad (2008), determina que el 55\% mantienen una frecuencia de relaciones sexuales diariamente $\mathrm{o}$ semanalmente, y el $42 \%$ restante mensual o esporádicamente, reflejándose un incremento en la frecuencia de las relaciones coitales respecto al cuarto sondeo de opinión de la gente joven del 2003, que refiere un $45 \%$ de relaciones sexuales diarias o semanalmente, y el $51 \%$, con relaciones sexuales mensuales o esporádicamente, reafirmando la evolución en ambas muestras de nuestro trabajo.

Sueiro y Diéguez (2001), indican que el 39.0\% mantuvieron una frecuencia de coitos, en el último año, de menos de 1 vez al mes, y un $26.7 \%$ de los mismos, de 1 a 3 veces al mes, resultados semejantes a los encontrados con los varones del curso 1997-98.

Si nos centramos en el número de parejas coitales que tuvieron durante el último año en que iniciaron sus coitos, el porcentaje más elevado se observa en una 
pareja (el 64.3\%, de los varones del curso 1997-98, y el $62.5 \%$, para el curso 2007-08), por el contrario, el número de estudiantes que tuvieron más de una pareja coital es de un $28.7 \%$, en la primera muestra, y del $29.8 \%$, en la segunda, no encontrando cambios significativos después de 10 años.

Sueiro y Diéguez (2001), señalan que el 58.1\% sólo tuvo una pareja coital, un $14.3 \%$ tuvieron dos parejas coitales, y para el 19.2\% 3 ó más parejas, porcentaje inferior al obtenido con los varones del curso 1997-98; Teva et al. (2009b), determinan que el número medio de parejas sexuales en el varón es de 3.2.

Examinando los estudios del IJEs, se observa, en el IJE-2000, que el 74.0\% de los varones sólo tuvieron una pareja, un $10.0 \%$ dos parejas, un $5.0 \%$ tres parejas, y el $6.0 \%$ cuatro ó más parejas en el último año, resultados que se aproximan a los de la muestra de varones del curso 1997-98; en el IJE-2004, se constata que un $62.4 \%$ sólo tuvieron una pareja, el $14.1 \%$ dos parejas, un $7.0 \%$ tres parejas, y el $11.0 \%$ cuatro ó más, datos que confirman los obtenidos con la muestra de varones del curso 2007-08; el IJE-2008, refiere que un $61.0 \%$ sólo tuvieron una pareja, el $14.1 \%$ dos parejas, un $8.3 \%$ tres parejas, y el $12.3 \%$ tuvieron cuatro ó más, resultados que también coinciden con los de la muestra de varones del curso 2007-08. A su vez, en la encuesta internacional DUREX-2004, se señala que un $61.1 \%$ de los hombres sólo tuvieron una pareja, el $14.1 \%$ dos parejas, un $8.3 \%$ de los varones tres parejas y el $12.1 \%$ cuatro ó más parejas coitales.

Por su parte, la $2^{\mathrm{a}}$ Encuesta Schering de Sexualidad y Anticoncepción en la Juventud Española (2005), reseña que, aquellos que han mantenido relaciones coitales en los últimos 12 meses, el $43.3 \%$ mantiene la misma pareja sexual más de 6 meses, porcentaje inferior al obtenido respecto a los varones del curso 2007-08.

Respecto a la consecución del orgasmo en el último coito en los hombres, el $86.4 \%$, del alumnado del curso 1997-98, y un 84.2\%, del curso 2007-08, manifestaron sentirlo, sin producirse cambios significativos después de 10 años. De este modo, Sueiro y Diéguez (2001), señala que el $88.6 \%$ manifiestan que sintieron orgasmo en el último coito, porcentaje superior al encontrado en el curso 1997-98. Por su parte, Lasheras et al. (2005), destacan que el $84 \%$ de los varones expresan satisfacción con orgasmo, porcentaje ligeramente inferior al encontrado con los varones del curso 2007 08.

Atendiendo al consumo de alcohol en el último coito por parte propia o de la pareja, se observa un incremento en el consumo de alcohol en los 10 años, con un $27.8 \%$, en el curso $1997-98$, y del $32.4 \%$, para 2007-08, apreciándose cambios significativos en esta conducta. Teva et al. (2009b), manifiestan que, en el último coito, el $24.0 \%$ de los varones consumieron drogas frente a un $76 \%$ que no la utilizaron, porcentajes similares al del curso 2007-08.
Si nos referimos a la utilización de anticoncepción en el último coito, se manifiesta un incremento significativo en su uso en al alumnado del curso 200708 respecto al del curso $1997-98$ (84.9\% y 86.9\%, respectivamente). Gras et al. (2000), refieren que menos de la mitad de los hombres utilizan siempre el preservativo. También Lameiras et al. (2007-08), afirmaban que el $97.0 \%$, de los activos sexualmente, utilizan algún método anticonceptivo en sus relaciones sexuales, porcentaje superior a los del curso 2007-08.

Vinaccia et al. (2007), entre adolescentes, destaca que solo un $34.6 \%$ utilizan siempre el preservativo en el coito vaginal, un $35.7 \%$ en el coito anal, un $27.7 \%$ en las relaciones con la pareja estable, y un $58.1 \%$ en las relaciones esporádicas. Por tanto, las distintas investigaciones consultadas revelan porcentajes inferiores a los encontrados con las muestras del curso $1997-98$ y $2007-08$, encontrando una gran diversidad de utilización de los mismos, siendo a que están influyendo diferentes variables sociales como: cultura, nivel económico, religión, edad, accesibilidad, entre otras, que mediatizan su uso.

Si nos centramos en el tipo de método anticonceptivo utilizaron en el primer coito, la gran mayoría usa el preservativo (el $81.7 \%$, del curso $1997-98$ y del $79.4 \%$ del 2007-08), seguido de los hormonales (11.3\% y $15.3 \%$, respectivamente), del coito interrumpido $(2.4 \%$ y $1.7 \%$, respectivamente), sin encontrar presencia en los otros métodos. No se aprecian cambios significativos después de 10 años de evolución.

En el IJE-2004 constatan que el uso mayoritario de anticoncepción corresponde al preservativo en un $84.7 \%$, seguido de la píldora en un $12.9 \%$, el coito interrumpido $(0.2 \%)$, y los otros métodos apenas tienen presencia (1.1\%); en el IJE-2008, destacan que el porcentaje mayoritario corresponde a la utilización del preservativo en un $83.4 \%$, seguido de la píldora en un $15.6 \%$, el coito interrumpido $(0.3 \%)$ y con un $0.7 \%$ los otros métodos. Si se comparan estos datos se aprecian diferencias escasas, y se deben básicamente a la irrupción de los métodos hormonales. Resultados estos que están en la línea de los encontrados con el grupo de universitarios del curso 2007-08, en el cual el porcentaje mayoritario se corresponde a la utilización del preservativo en un $79.4 \%$, seguido de la píldora en un $15.3 \%$, los otros métodos apenas tienen presencia. Estas cifras no son extrañas y, aparte de coincidir con las IJE2008 , se ajustan con la comparativa internacional en la que España es el país del mundo en el que más se utiliza el preservativo como método anticonceptivo y para prevenir las E.T.S. Nuestros resultados referidos al curso 2007-08 no coinciden con los hallados por los del IJE, lo cual puede ser debido a que a nuestra muestra le preguntamos por el último coito y, los estudios del IJE, indagan por el uso habitual del método anticonceptivo adoptado en sus coitos. 
Por su parte, Lameiras et al. (2007-08), apuntan que el $56.9 \%$ de los varones usan siempre o casi siempre el preservativo (porcentaje es inferior a los varones del curso 2007-08) y un 27.0\% utiliza siempre o casi siempre la píldora (porcentaje superior al del curso 2007-08).

En torno a un $94 \%$, de cada una de las muestras manifiestaron mo haber tenido problemas con el uso del último mecanismo de protección y alrededor del 5.0\%, de ambas muestras, sí los tuvieron, no encontrando cambios significativos después de 10 años. También Sueiro y Diéguez (2001), manifiestan que el 95.2\% de los varones no tuvieron problemas con el uso de la anticoncepción en el último coito, datos estos semejantes a los encontrados con la muestra del chicos del curso 1997-98. Finalmente, respecto a quien propuso el uso de la anticoncepción en el último coito, el $68.8 \%$, de los varones del curso $1997-98$, y el 70.3\%, del curso 2007-08, registran que fueron ellos mismos, y solamente un $11.7 \%$, de los primeros, y un $5.3 \%$, de los segundos, fue una decisión conjunta, encontrando cambios significativos después de 10 años.

A tenor de los resultados hallados, observamos cómo la educación sexual existente hasta este momento no parece suficiente para la reducción de los riesgos en este ámbito de la vida.

\section{Referencias}

Cleland, J. y Ali, M.M. (2006). Sexual abstinence, contraception, and codom use by Young African women: a secondary analysis of survey data. The Lancet, (368), 1788-1793.

Comas, D. (2008). Informe de la juventud en España. Madrid: Ministerio de Trabajo y Asuntos Sociales.

Comas, D., Aguinaga, J., Orazo, F., Espinosa, M.A., y Ochaita, F. (2003). Jóvenes y estilos de vida. Valores y riesgos de los jóvenes urbanos. Madrid: FAD

Cortés, A., García, R., Monterrey, P., Fuentes, A., y Pérez, D. (2000). Sida, Adolescencia y Riesgos. Revista Cubana Medicina General Integral, 16 (3), 253-260.

Encuesta Durex 2004. Recuperado el 10 de Septiembre de 2009, de http://www.durex.com/default. asp.

Failde, J.M., Lameiras, M., y Bimbela, J.L. (2008). Prácticas sexuales de chicos y chicas españoles de 1424 años de edad. Gaceta Sanitaria, 22 (6), 511-519.

Gras, M.E., Planes, M., Soto, J., y Font-Mayolas, S. (2000). Percepción de riesgo y comportamientos heterosexuales relacionados con el SIDA: estudio comparativo con cinco muestras de universitarios. Cuadernos de Medicina Psicosomática y Psiquiatría de Enlace, (54), 39-45.

Gujral, L.M. y Barreto, A. (2004). Sexual activity knowledge and condom use among youth in Mozambique. International Conference on AIDS. Jul 11-16, Abstract no. TuPeC4791.
Injuve, (2000). Informe de la Juventud en España 2000. Madrid: Ministerio de Trabajo y Asuntos Sociales.

Injuve, (2004). Informe Juventud en España (2004). Madrid: Ministerio de Trabajo y Asuntos Sociales

Injuve, (2008). Informe Juventud en España (2008). Madrid: Ministerio de Trabajo y Asuntos Sociales.

Lameiras, M., Nuñez, A.M., Carrera, M.V., y Rodríguez, Y. (2007/2008). Conducta sexual y uso del preservativo masculino en muestra de jóvenes universitarios Gallegos. Cuadernos de Medicina Psicosomática y Psiquiatría de Enlace, (84-85), 49-56.

Lasheras, M.G., Cuñe, J., Bautista J., y Farré J.M. (2005). Hábitos sexuales en jóvenes universitarios. Cuadernos de Medicina Psicosomática y Psiquiatría de Enlace, (74), 57-63.

López, F. (1990). Educación sexual. Madrid: Fundación Universidad Empresa.

Ochaita, E. y Espinosa, M.A. (2003). Las prácticas sexuales de los adolescentes y jóvenes españoles. Estudios de la juventud, (63), 49-62.

Rodríguez, J. y Traverso, C.I. (2007). Conductas y Educación sexual en universitarios españoles. Málaga: Grupo Editorial.

Segunda Encuesta Schering Sexualidad y Anticoncepción en la Juventud Española (2005). Recuperado el 23 de Enero de 2008, de http://www.equipodaphane.es.

Sueiro, E. y Diéguez J.L. (2001). Juventud y sexualidad (pp. 257-269). Actas do VI Congreso GalaicoPortuguês de Psicopedagogía. Braga

Teva, I., Bermúdez, M.P., y Buela-Casal, G. (2009b). Conductas de riesgo para la infección por el VIH y las enfermedades de transmisión sexual (ETS) en adolescentes en el año 2007: diferencias en función de variables sociodemográficas. Revista Española de Salud Pública, (83), 309-320.

Vinaccia, S., Quiceno, J. M., Gaviria, A. M., Soto, A.M., Gil, M.D., y Ballester, R. (2007). Conductas Sexuales de Riesgo para la Infección por VIH/SIDA en Adolescentes Colombianos. Terapia psicológica, 25 (1), 39-50. 